\title{
DIAGNOSTICS OF MODELS FOR COSMIC STRUCTURE FORMATION
}

\author{
Masataka Fukugita \\ Yukawa Institute for Theoretical Physics, Kyoto University \\ Kyoto 606, Japan
}

In this talk I shall discuss two topics related to modeling of cosmic structure formation. In the first part I shall briefly review the problem of cosmological parameters, which are crucial for any of model calculations. An emphasis is made on whether we need a non-zero cosmological constant. In the latter part cosmic microwave background (CMB) anisotropies are discussed as a probe of the models for cosmic structure formation.

\section{Cosmological Parameters}

The Freedmann universe is described by three fundamental parameters, the Hubble constant $H_{0}$, the mass density of the universe $\Omega_{0}=\rho / \rho_{\text {crit }}$ and the cosmological constant $\Lambda$. Most of theoretical calculations for evolution of cosmic structure adopt $H_{0}=50 \mathrm{~km} \mathrm{~s}^{-1} \mathrm{Mpc}^{-1}$ and $\Omega_{0}=1$ as a "theoretical prejudice". In the following. I discuss if they are supported observationally. I use the normalisation $H_{0}=100 \mathrm{~km} \mathrm{~s}^{-1} \mathrm{Mpc}^{-1}, \Omega_{0}=\rho / \rho_{\text {crit }}$ and $\lambda_{0}=\Lambda / 3 H_{0}^{2}$, so that $\Omega_{0}+\lambda_{0}=1$ for the flat universe.

1. Hubble constant

Accumulating observational evidence based on distance ladders points towards the high value $H_{0}=75-100 \mathrm{~km} \mathrm{~s}^{-1} \mathrm{Mpc}$. The low value $H_{0}=40-60 \mathrm{~km} \mathrm{~s}^{-1} \mathrm{Mpc}^{-1}$ now seems rather unlikely from the observational ground.

The Cepheid work which has been carried out over the last five years determined the distance to the local calibrators up to the M81 group to within $\sim 15 \%$ or so (e.g., Tammann 1987, Freedmann 1990). The Cepheid distance to the LMC is confirmed by the $u v$ light curve from the ring echo of SN1987A (Panagia 1991). This eliminates a doubt about the Cepheid zero point based on distance ladders. The uncertainty of the local calibrator distance ( $\sim 0.65 \mathrm{mag})$ as was discussed in Aaronson and Mould (1983) does not seem to exist any more.

The most important progress made recently is the discovery of new techniques using planetary nebula luminosity functions (Jacoby et al. 1990) and surface bright- 
ness fluctuations (Tonry et al. 1990), which enable us to measure the distance to individual galaxies near the Virgo centre. Both methods, when applied to the local calibrators, yield the distance relative to M31 in excellent agreement with the Cepheid distance. For galaxies in a distance of $10-15 \mathrm{Mpc}$, these two methods and the Tully-Fisher (TF) method all give the answer convergent to $0.2-0.3 \mathrm{mag}$, i.e., $\$ 15 \%$ in the distance (e.g., Tonry 1991). The distance to the Virgo centre with these methods is $15 \pm 1 \mathrm{Mpc}$ in agreement with the Pierce-Tully (1989) distance by the $I$-band TF method. This leads to $85 \pm 10 \mathrm{~km} \mathrm{~s}^{-1} \mathrm{Mpc}^{-1}$ insensitive to the choice of the infall model and the recession velocity used. A similar conclusion is also derived for other clusters at similar distances (Tonry 1991). We remark that the distance by the intermediate indicators used more traditionally, such as globular cluster luminosity functions and novae etc., correlates poorly with other standards.

A direct link between the local calibrators and the Coma cluster by the TF method also gives $H_{0}=92 \pm 15 \mathrm{~km} \mathrm{~s}^{-1} \mathrm{Mpc}^{-1}$ after a correction for the Malmquist bias (Fukugita et al. 1991a). A similar value is also obtained from the Hubble diagram of the SNela, after new zero point calibrations (Fukugita and Hogan 1991).

There is a recent work which indicates a low value of $H_{0}$ from the differential time delay of gravitational lensing $0957+561$ (Roberts et al. 1991). We can not yet take this value too seriously because of substantial uncertainties, especially in the velocity dispersion to be put into the lensing equation (e.g., a typical $3 / 2$ factor, see Turner et al. 1984).

If $H_{0} \geq 75 \mathrm{~km} \mathrm{~s}^{-1} \mathrm{Mpc}^{-1}$ a cosmic age of $t_{0} \leq 13 \mathrm{Gyr}$ is concluded for the Freedmann Universe even for $\Omega_{0}=0$. If $t_{0} \approx 15 \mathrm{Gyr}$ as inferred from the globular cluster aging, a non-zero $\Lambda$ is necessary. This may be the most "compelling" evidence for a non-zero $\Lambda$.

\section{Number Count of Faint Galaxies}

The Tyson's $B$-band counting (Tyson 1988) exhibits a substantial excess of the number of galaxies at faintest (25-27 $\left.B_{J} \mathrm{mag}\right)$ magnitudes. This was then interpreted as evidence in favour of non-zero $\Lambda$ (Fukugita et al. 1990a). Similar excess is also seen in the $R$ and $I$ band counts (Tyson 1988), and his data are fitted well with non-zero $\Lambda$ with canonical evolution models of galaxies (Fukugita et al. 1991c).

The interpretation of the $K$-band counting (Cowie et al. 1990, 1991) is confusing. Cowie argued that the $\Omega_{0}=1$ model explains their data. On the other hand other authors (Fukugita et al. 1991c) concluded that their data are fitted best with $\Omega_{0}=0.1, \lambda_{0}=0 ; \Omega_{0}=1$ is disfavoured and $\Omega_{0}=0.1, \lambda_{0}=0.9$ is not excluded. The argument which has often been made that the $K$-band count is more reliable to test cosmology, i.e., not sensitive to evolution, is misleading: While the K-band counting has the advantage that it is less affected by transient phenomena such as the burst of star formation, it is indeed sensitive to the old population. If stars form gradually in the first Gyrs, as in the case when gas infall plays an important role, galaxies are fainter in the $K$-band than are expected from the galaxy today 
in the closed model. Therefore, at least two possibilities remain to give a consistent solution: (i) $\Lambda \neq 0$ and a small $\Omega_{0}$, with the canonical evolution model (with some corrections for increasing old populations for the $K$-band count (Fukugita et al. 1991c) (ii) $\Lambda=0$, with the presence of a new population of galaxies (presently dwarf), which accounts for the excess count in the $B, R, I$ bands (Cowie et al. 1991). It can be shown that a simple merging hypothesis (Guiderdoni and RoccaVolmerange 1990) does not save the $\Omega=1$ model, if selection effects at the Tyson's observation are taken into account (Yoshii and Fukugita 1991).

\section{Gravitational Lensing}

It has been pointed out that gravitational lensing frequency is sensitive to the cosmological constant (Fukugita et al. 1990a, Turner 1990). This effect is further explored by a few authors (Fukugita and Turner 1991; Kochanek 1991a,b; Fukugita et al. 1991b). A reasonably realistic calculation shows that 11-35 lenses are expected for 4250 quasars in the Hewitt-Burbidge catalogue if $\Omega_{0}=0.1$ and $\lambda_{0}=0.9$. This is compared with $3-7$ for $\Omega_{0}=0.1, \lambda_{0}=0$ and $2-5$ for $\Omega_{0}=1, \lambda_{0}=0$. This prediction assumes that the catalogue does not miss lenses giving multiple images with an angular separation $\theta>2^{\prime \prime}$. The number of lens candidates varies from 4 to 9 depending upon how to count (Fukugita and Turner 1991). If we assume that the catalogue does not miss more than $1 / 3$ of lenses with $\theta>2^{\prime \prime}, \Omega_{0}=0.1, \lambda_{0}=0.9$ is marginally allowed, and $\lambda_{0}>0.95$ is disfavoured for the flat universe.

To make the statistical lens test useful, we need in general a homogeneous lens survey with more than $10^{3}$ quasars. A sample with a size of $>10^{2}$ would give a meaningful result, if a survey is made for bright and moderately high $z$ quasars. The snap shot survey of Bahcall et al. (1991), would bring interesting information on the $\Lambda$ problem if the sample size could be tripled; an upper limit $\lambda_{0} \lesssim 0.8$ would be derived at $90 \%$ C.L., if they would not see any lenses with a tripled-size sample.

4. Other $\Lambda$ tests

There are several other tests for $\Lambda$ discussed in the literature. They are, however, even less convincing or do not give a direct probe for $\Lambda$.

(i) Damped Ly $\alpha$ frequencies. Lanzetta (1991) observed at least 32 damped Ly $\alpha$ systems for a redshift interval $\Delta z=161$. He argued that the predicted number $10 \pm 3$ in the $\Omega_{0}=1$ geometry, under the assumption that the damped Ly $\alpha$ system is normal galaxies, is substantially less than is observed. He also noted that the $\Omega_{0}=0.1$ geometry does not help much (15 \pm 5$)$. If $\lambda_{0}=0.9$ and $\Omega_{0}=0.1$, however, we expect $29 \pm 9$, consistent with the observed number. This may be taken as evidence in favour of a non-zero $\Lambda$.

(ii) Ly $\alpha$ cloud number density (Fukugita and Lahav 1991, Turner and Ikeuchi 1991). The redshift distribution $N(z)$ of the line of sight number density of Ly $\alpha$ clouds at low redshift is sensitive to $\Lambda$. If we parametrise $N(z)=(1+z)^{\gamma}, \gamma$ takes a value between 0.5 and 1 for the $\Lambda=0$ universe for no evolution of the cloud. With $\lambda_{0}=0.9$ 
and $\Omega_{0}=0.1$, however, this index $\gamma$ becomes 1.4 for the redshift interval $z=0$ to 2 ( $\gamma$ is insensitive to $\Lambda$ at higher $z, z \gtrsim 2$ say). For $z>2$ observations yield $\gamma \simeq 2-2.5$, which is evidence for strong evolution of Ly $\alpha$ clouds. The recent report from the Hubble Space Telescope gives $\gamma=0.8 \pm 0.4$ between $z=0$ and 2 (Morris et al. 1991). They concluded that this suggests that evolution ceases towards low redshift. This interpretation will meet a trouble if $\lambda_{0} \gtrsim 0.8$; with a high $\lambda_{0}$ we have to think about the situation that comoving number density of Ly $\alpha$ clouds increases at a low $z$, which is opposite to what happens at high $z$.

(iii) Peculiar velocity-acceleration relation $\left(v \propto \Omega_{0}^{0.6} g\right)$. Peebles (1984) showed that non-zero $\Lambda$ makes a negligible effect on the clustering dynamics. This is also true for high-redshift clusters (Lahav et al. 1991); an apparent $\Lambda$ effect cancels in observable quantities.

(iv) Angular correlation function $w(\theta)$ in the CDM universe. Efstathiou et al. (1990) showed that $\Omega_{0}=1$ CDM models do not exhibit a sufficient power at a large angle as observed in the APM survey. They obtained, however, a good fit with $\Omega_{0}=0.2$ $(h=1.0)$. If one imposes that the universe is flat, $\Omega_{0}+\lambda_{0}=1$, this implies $\lambda_{0}=0.8$.

(v) Galaxy clustering at $z \approx 2$ (Tyson's counting). Efstathiou et al. (1991) claimed that the data at high $z$ require too rapid clustering of galaxies towards $z=0$ to reconcile with the theory for $\Omega_{0}=1$. They have argued that this problem is alleviated if $\Omega_{0} \approx 0.1$. For the flat universe, this means $\lambda_{0} \approx 0.9$.

\section{Small Angular Scale CMB Anisotropies}

A tight connection exists between spatial temperature fluctuations of CMB and cosmic structure observed today. An important point is that the effect involves very simple physical processes and there is little uncertainty, at least in principle, in predicting this connection. Therefore, small angular scale CMB fluctuations give an important constraint on the model of large-scale structure formation. In the $\Lambda=0$ cosmology the angle of the beam throw $\theta$ is related to the comoving length scale $\ell$ as $\ell=17.4 \mathrm{Mpc}\left(\theta / 10^{\prime}\right) \Omega_{0}^{-1}$.

\section{Principles of calculations}

The initial condition of the calculation is set by giving power spectrum fluctuations to the Fourier component of $\delta \rho / \rho$, as $\left|\delta_{k}\right|^{2}=A k^{n}$. Usually the phases of the Fourier modes are assumed to be random and Gaussian. The perturbations are divided into two classes; adiabatic, in which all components fluctuate in the same way, and isocurvature, where the perturbations do not couple to the curvature. Adiabatic perturbations are usually regarded as a more natural possibility from the particle physics viewpoint. The growth of the perturbations before recombination is calculated in a single viscous fluid, in which photons tightly couple to matter. After recombination starts, the collisional Boltzmann equation is solved with Thomson scattering taken into account explicitly. After the Thomson optical depth becomes sufficiently smaller than unity, the photon propagation is approxi- 
mated by free streaming, and the growth of matter perturbations is computed by the linear regime to predict $\delta \rho / \rho$ today. The whole calculation should then be valid for a comoving scale larger than $\sim 8 h^{-1} \mathrm{Mpc}$ where $[\delta \rho / \rho]_{0} \lesssim 1$. The normalisation $A$ is fixed so that the matter fluctuations give large-scale structure as characterised by two-point correlation functions of the galaxy distribution at the present epoch, if light traces mass. The biasing parameter $b \neq 1$ is often introduced, however, to relate the galaxy distribution to the mass distribution that we need.

We then calculate the two point correlation function $C(\theta)=\left\langle\delta T\left(\hat{\gamma}_{1}\right) / T, \delta T\left(\hat{\gamma}_{2}\right)\right.$ $|T\rangle$ with $\cos \theta=\hat{\gamma}_{1} \cdot \hat{\gamma}_{2}$ between the two directions $\hat{\gamma}_{1}$ and $\hat{\gamma}_{2}$. $\delta T$ is given by the sum of surface brightness fluctuations at the last scattering surface, which refer directly to matter fluctuations, and the potential energy difference between the last scattering surface and the present epoch. The velocity field at the last scattering surface also contributes to $\delta T$. The fluctuations in the potential are referred to as the Sachs-Wolfe effect. The contribution from surface brightness fluctuations is important only when the physical distance between the two positions pointed by $\hat{\gamma}_{1}$ and $\hat{\gamma}_{2}$ is smaller than the horizon, which corresponds to $\theta_{H}=1.8 \Omega_{0}^{1 / 2}$ in the $\Lambda=0$ cosmology. The Sachs-Wolfe contribution dominates $C(\theta)$ for $\theta \gtrsim \theta_{H}$, and it is still non-negligible $(\sim 20-30 \%)$ even at a $10^{\prime}$ scale (e.g., see Fig. 2 of Fukugita et al. 1990b). $C(\theta)$ generally decreases as $\theta$ increases, and takes approximately the form $C(\theta)=\left[1+\left(\theta / \theta_{c}\right)^{\beta}\right] C(0)$ with $\beta=1.5-2.5$ and $\theta_{c}$ of the order of $10^{\prime}$. The CMB anisotropies most often quoted by experimentalists are the rms value,

$$
\Delta T / T \equiv\left\langle\left(T\left(\hat{\gamma}_{1}\right)-T\left(\hat{\gamma}_{2}\right)\right)^{2}\right\rangle^{1 / 2} /\langle T\rangle=\sqrt{2}[C(0)-C(\theta)]^{1 / 2}
$$

where $C(\theta)$ for actual experiments must be smeared by the beam width $\sigma$. With given $n, H_{0}, \Omega_{0}, \Omega_{B}$ and $\lambda_{0}$ the predicted $\Delta T / T$ is compared with the experimental limits to test the model.

From (1) it is clear that $\Delta T / T$ at a large $\theta$ does not give much information on $C(\theta)$, for $\Delta T / T$ is simply dominated by $C(0)(\gg C(\theta))$ as $\theta \rightarrow \infty$. This is particularly true for a small beam size experiment. There is always an optimal $\theta$ and $\sigma$ for a given model to test it against the experiment. Bond et al. (1991) formulated this nicely in terms of a harmonic expansion of $C(\theta) ; C(\theta)=\sum(2 \ell+$ 1) $C_{\ell} P_{\ell} \simeq \int d(\ln \ell) \ell^{2} C_{\ell} P_{\ell}$. With an actual experiment a window function $F_{\ell}=$ $\left(1-P_{\ell}\right) \exp \left(-\ell^{2} \sigma^{2}\right)$ is to be multiplied in the integrand. When the model power $\ell^{2} C_{\ell}$ and the experimental power $F_{\ell}$ match as a function of $\ell$, the experimental command becomes maximal. Bond et al. have shown, for example, that $\theta \sim 1^{\circ}, \sigma \sim$ 0.5 is optimal to test the CDM model. With this formalism Efstathiou (1991) demonstrated in a clear manner that triple beam experiments loose information significantly compared with the double beam case, whereas a background can be suppressed by double switching.

2. Result

It is now well-known that the absence of anisotropy at 4.5 (Uson and Wilkinson 
1984) and 7'.15 (Readhead et al. 1987; OVRO experiment) down to a level of $\Delta T / T \sim$ a few $\times 10^{-5}$ essentially rules out all models in which present cosmological mass density is composed entirely of baryons (including the case with $\Lambda \neq 0$ ); see, Kaiser and Silk 1986. Two interesting remaining possibilities are CDM models and isocurvature baryon models with reheating. In both cases strong constraints are derived on the model from $\Delta T / T$.

(i) Case of the CDM model. Calculations are done mainly by three groups, Vittorio and Silk 1984, 1985, Vittorio et al. 1991; Bond and Efstathiou (BE) 1984, 1987, Bond et al. 1991, Efstathiou 1991 and a Japanese group (Sugiyama 1989, Fukugita et al. 1990b, Suto et al. 1990, Sugiyama et al. 1990, Fukugita and Sugiyama 1991) with three different gauge choices. The first two groups adopt $h=0.5$, the theorists' favourite. BE further assumed $\Omega_{0}=1$ for most of their calculations. While the principle is simple, actual calculations are a little complicated. We observe that $\mathrm{BE}$ and Japanese agree within $\lesssim 10 \%$ for $\Delta T / T$, but Vittorio, Silk and their collaborator's result is off from the other two by $20-50 \%$ (see Table I). This disagreement, however, does not seem too serious to extract physics conclusions at the level of accuracy required for time being.

Table I. Comparison of CMB anisotropy calculations for $\Omega_{0}=1, \Omega_{B}=0.03$ and $h=0.5$.

\begin{tabular}{|c|c|c|c|c|}
\hline & \multirow{2}{*}{ normalisation* } & \multicolumn{2}{|c|}{$\Delta T / T\left(\times 10^{5}\right)$} & \multirow{2}{*}{$C(0)\left(\times 10^{10}\right)$} \\
\hline & & $4^{\prime} .5$ & $7^{\prime} .5$ & \\
\hline BE84 & $J_{3}$ & $0.42^{* *}$ & - & $7.8^{* *}$ \\
\hline $\mathrm{BE} 87$ & $J_{3}$ & 0.49 & 0.92 & 7.8 \\
\hline Holtzman 1989 & $J_{3}$ & 0.50 & 1.1 & 8.6 \\
\hline Fukugita et al. $1990 \mathrm{~b}$ & $J_{3}$ & 0.55 & 1.0 & 7.3 \\
\hline Fukugita et al. 1990b & $\delta M$ & 0.58 & 1.06 & 7.7 \\
\hline Vittorio-Silk 1984 & $\delta M$ & 0.67 & 1.3 & 4.4 \\
\hline Vittorio et al. 1991 & $\delta M$ & 0.73 & 1.39 & - \\
\hline
\end{tabular}

* $J_{3}$ normalisation: $J_{3}(R=10 \mathrm{Mpc})=\int_{0}^{R} r^{2} d r \xi(r)=280 h^{-1}(\mathrm{Mpc})^{3} . \quad \delta M$ normali sation: $\left\langle(\delta M / M)^{2}\right\rangle=1$ at $R=8 \mathrm{Mpc}$.

** Sachs-Wolfe terms are not taken into account.

The basic result may be summarised as follows: (a) For $h=0.5$ and $\Omega_{0}=1$, the model is allowed without biasing $(b=1)$ if $\Omega_{B}<0.2$. A decrease of $\Omega_{B}$ further relaxes the constraint. Conversely, if we require $\Omega_{B}$ to lie in the range favoured by nucleosynthesis $\Omega_{B}=0.03-0.07, \Omega_{0}>0.6(>0.4)$ is concluded for $b=1(2)$. The existence of the lower limit on $\Omega_{0}$ is understood from the two effects: A decrease of $\Omega_{0}$ means an increase of the physical length scale at the last scattering surface for a. given $\theta$, which causes an increase of $C(0)-C(\theta)$, and the decrease of the growth 
rate of perturbations in a low density universe requires a large initial amplitude, both effects working to make the constraint from $\Delta T / T$ tighter. The constraint obtained from the new South-Pole experiment (Meinhold and Lubin 1991) at $\theta=1^{\circ}$ turns out to be quite similar to the one derived from the OVRO experiment. (b) With $h=1.0$ the constraints are substantially relaxed: For $\Omega_{B}=0.01-0.02$ which is favoured by nucleosynthesis, $\Omega_{0}>0.2-0.25$ is derived from the OVRO and the South-Pole experiments. This relaxation is readily understood by the fact that physical scale on the last scattering surface decreases as $H_{0}$ increases for a given $\theta$, leading to a decrease of $C(0)-C(\theta)$. (c) Addition of a non-zero $\Lambda$ generally relaxes the constraint. This can also be understood from the $\ell-\theta$ relation and the change of the growth rate in the presence of $\Lambda$. The constraints for the case of $\Omega_{0}+\lambda_{0}=1$ are generally stronger than those for the case of $\Omega_{0}=1, \lambda=0$, however. For $h=1$ models, $\Omega_{0} \gtrless 0.1$ with $\lambda_{0}=1-\Omega_{0}$ is allowed without biasing $(b=1)$ for the favoured range of $\Omega_{B}$ [For $h=0.5$ the low density model is marginal (need $b>2-3$ ) even with the cosmological constant that makes the universe flat: This case, however, is not important as the cosmic age becomes as long as 25Gyr.] (d) No strong constraints are derived from the limits on $\Delta T / T$ at few degree angular scales from the Tenerife experiments (Davies et al.1987; Lasenby et al. 1991).

The result discussed here is summarised in Table II in terms of the bound on the biasing parameter $b$. The favoured range for $b$ is $b=1-2.5$.

Table II. Constraints on the CDM model from $\triangle T / T$ (OVRO \& South Pole) represented in terms of the biasing parameter $b$. The preferred range of $b$ is $1-2.5$. The constraints are estimated from calculations available to date.

\begin{tabular}{|c|c|c|c|c|}
\hline & & $\Omega_{0}=1$ & $\Omega_{0}=0.2$ & $\Omega_{0}=0.2 \quad \lambda_{0}=0.8$ \\
\hline$H_{0}=50$ & $\Omega_{B}=0.03$ & $b \gtrsim 0.8$ & $b \gtrsim 4-5$ & $b \gtrsim 2^{*}$ \\
\hline$H_{0}=100$ & $\Omega_{B}=0.01$ & $b Z 0.4^{*}$ & $6 \gtrsim 1.5^{*}$ & $b \gtrsim 0.7$ \\
\hline
\end{tabular}

* The cosmic age constraint is not satisfied with these cosmological parameters.

(ii) Case for isocurvature models with reheating (Peebles 1987). Constraints from the OVRO and the South-Pole experiments are weak, since small-scale anisotropies ( $\theta \ll$ $8^{\circ} \Omega_{0}^{1 / 2}$ ) are substantially lessened by reheating (Kaiser 1984). Strong constraints, however, are derived from the Tenerife and COBE experiments (Smoot et al. 1991): Only the case with a steep power index $n \geq 3-4$ survives the constraints (Efstathiou and Bond 1987).

3. Effect of gravitational lensing on CMB anisotropies

Kashlinsky 1988 and Tomita 1988 suggested that gravitational lensing in the early Universe lessens small-scale CMB anisotropies. Cole and Efstathiou (1990), and Sasaki (1990) then pointed out that this is not true, since the two light rays 
separated by a small angle are deflected almost in the same way. They have shown that $\Delta T / T$ in a small anglular scale increases due to the lensing effect. This increase, however, is small and hardly modifies the prediction without lensing. This conclusion holds even in the presence of a large $\Lambda, \lambda_{0} \sim 0.9$, say (Fukugita et al. 1991b).

\section{Conclusion}

From purely observational view point a high $H_{0}$ and a low $\Omega_{0}$ are favoured, and the $\Lambda \neq 0$ cosmology seems to have more advantages than the $\Lambda=0$ cosmology, though $\Lambda \neq 0$ is not yet compelling. From the theory side no principles forbid the existence of $\Lambda \neq 0$, while it is thought not attractive as we do not understand why $\Lambda$ is so small. We do not understand either, however, why the matter density is so small compared with the gravity scale: Only philosophical reasoning for this smallness available at present is inflation. Inflation, however, only states that $\Omega_{0}+\lambda_{0}=1$. I feel at least it worth trying to play all games of cosmology models with a non-zero $\Lambda$ in the present circumstance.

CMB anisotropies place very stringent constraints on models of structure formation. We should stress that $\Lambda \neq 0$ alone does not solve the problem; we still need something unusual (CDM, reheating, etc.) to reconcile the model with observations.

I would like to thank Dick Bond, Len Cowie, Ofer Lahav and Nicola Vittorio for discussions. I am also grateful to the Yamada Science Foundation for the support.

\section{References}

Aaronson, M. and Mould, J. 1983, Astrophys. J. 265, 1.

Bahcall, J. et al. 1991, Astrophys. J., in press.

Bond J.R. and Efstathiou, G. 1984, Astrophys. J. 285, L45.

$$
\text { . 1987, MNRAS 226, } 655 .
$$

Bond, J.R. et al. 1991, Phys. Rev. Lett. 66, 2179.

Cole, S. and Efstathiou, G. 1989, MNRAS 239, 195.

Cowie, L.L. et al. 1990, Astrophys. J. 360, L1. . 1991, preprint.

Davies et al. 1987, Nature 326, 462.

Efstathiou, G. 1991, Oxford preprint.

Efstathiou, G. and Bond, J. R. 1987, MNRAS 227, 33p.

Efstathiou, G., Sutherland, W. J. and Maddox, S. J. 1990, Nature 348, 705.

Efstathiou, G. et al. 1991, Astrophys. J. 380, L47.

Freedmann, W.L. 1990, Astrophys. J. 335, L35.

Fukugita, M., Futamase, T. and Kasai, M. 1990a, MNRAS 246, 24p.

Fukugita, M. and Hogan, C. J. 1991, Astrophys. J. 368, L11.

Fukugita, M. and Lahav, O. 1991, MNRAS in press.

Fukugita, M. , Sugiyama, N. 1991, in preparation.

Fukugita, M. , Sugiyama, N. and Umemura, M. 1990b, Astrophys. J. 358, 28.

Fukugita, M. and Turner, E.L. 1991, MNRAS in press. 
Fukugita, M. et al. 1990c, Astrophys. J. 361, L1.

Fukugita, M. et al. 1991a, Astrophys. J. 376, 8.

Fukugita, M. et al. 1991b, Princeton preprint POP-422.

Fukugita, M. et al. 1991c, in preparation.

Guiderdoni, B. and Rocca-Volmerange, B. 1990, Astr. Astrophys. 227, 362.

Holtzman, J. A. 1989, Astrophys. J. Supple 71, 1.

Jacoby et al. 1990, Astrophys. J. 356, 332.

Kaiser et al. 1984, Astrophys. J. 282, 374.

Kaiser and Silk, J. 1986, Nature 324, 529.

Kashlinsky, A. 1988, Astrophys. J. 331, L1.

Kochanek, C.S. 1991a, Astrophys. J. in press.

1991 b, preprint.

Lahav, O. et al. 1991, MNRAS 251, 128.

Lasenby, A.N. et al. 1991, in Observational Tests of Inflation, in press.

Lanzetta, K. 1991, talk given at Aspen Winter Physics Conference.

Meinhold, P. and Lubin, P. 1991, Astrophys. J. 370, L11.

Morris, S. L. et al. 1991, Astrophys. J. 377, L21.

Panagia, N. 1991, in these proceedings.

Peebles, P.J.E. 1984, Astrophys. J. 284, L439. 1987, Astrophys. J. 315, L73.

Pierce, M.J. and Tully, R.B. 1988, Astrophys. J. 330, 579.

Readhead, A.C.S. et al. 1987, Astrophys. J. 346, 566.

Roberts, D. H. et al. 1991, Nature 352, 43.

Sasaki, M. 1989, MNRAS 240, 415.

Smoot, G.F. et al. 1991, Astrophys. J. 371, L1.

Sugiyama, N. 1989, Prog. Theor. Phys. 81, 1021.

Sugiyama, N., Gouda, N. and Sasaki, M. 1990, Astrophys. J. 365, 432.

Suto, Y. et al. 1990, Astrophys. J. Supple. 74, 665.

Tammann, G.A. 1987, in IAU Symposium 124, Observational Cosmology, p.151.

Tomita, K. 1988, Publ. Astr. Soc. Japan 40, 751.

Tonry, J.L. 1991, Astrophys. J. 373, L1.

Tonry, J.L., Ajhar, E.A. and Luppino, G.A. 1990, Astrophys. J. 346. L57.

Turner, E.L. 1990, Astrophys. J. 365, L43.

Turner, E.L. and Ikeuchi, S. 1991, Princeton preprint POP-433.

Turner, E.L., Ostriker, J. P. and Gott III, J. R. 1984, Astrophys. J. 284, 1.

Tyson, J.A. 1988, Astron. J. 96, 1.

Uson, J. M. and Wilkinson, D. T. 1984, Astrophys. J. 277, L1.

Vittorio, N. and Silk, J. 1984, Astrophys. J. 285, L39.

1985, Astrophys. J. 297, L1.

Vittorio, N. et al. 1991, Astrophys. J. 372, L1.

Yoshii, Y. and Fukugita, M. 1991, in preparation. 